Notre Dame Journal of Formal Logic

Volume XVIII, Number 1, January 1977

NDJFAM

\title{
A NOTE ON THOMASON'S REPRESENTATION OF S5
}

\author{
SATOSHI MIURA and SHIGEO ŌHAMA
}

Introduction S. K. Thomason has proved in [3] that a formula is provable in S5 iff all its substitution instances are in $H$, which is a unique correct set and is $\operatorname{Thm}(\boldsymbol{(})$. In order to prove this, he semantically showed that a formula $A\left(x_{1}, \ldots, x_{n}\right)$ is valid in S5 (tautology of S5 in the sense of Kripke [2], pp. 11ff.) iff $V^{*}\left(A\left(B_{1}, \ldots, B_{n}\right)\right)=1$ for all $B_{1}, \ldots, B_{n}$ in $\mathcal{L}_{c}$ (modal language with proposition constants).

In this paper, we shall show by means other than Kripke's model that $A\left(x_{1}, \ldots, x_{n}\right)$ is provable in S5 iff $\mu^{*}\left(A\left(B_{1}, \ldots, B_{n}\right)\right)=1$ for all classical formulas (without modal symbols), $B_{1}, \ldots, B_{n}$, for all $\mu^{*}$, where $\mu^{*}$ is essentially the same as $V^{*}$ above, except that $\mu^{*}$ is a valuation for modal formulas with proposition variables. In the last section of this paper, we shall also show a relation between Kripke's partial truth tables and $\mu^{*}$-valuations.

1 Formulation of $\mathrm{S} 5$ and truth valuation We prepare a countable set of proposition variables, $\Pi$, logical connectives, $\vee, \sim, \square$, and parentheses, (, ). Formulas are defined as usual. For any formulas $A$ and $B$, we define $A \wedge B$ as $\sim(\sim A \vee \sim B), A \rightarrow B$ as $\sim A \vee B, A \leftrightarrow B$ as $(A \rightarrow B) \wedge(B \rightarrow A)$, and $\diamond A$ as $\sim \square \sim A$. If $A$ and $B$ are formulas, the following expressions are axioms:

(A1) $(A \vee A) \rightarrow A$.

(A2) $B \rightarrow(A \vee B)$.

(A3) $(A \vee B) \rightarrow(B \vee A)$.

(A4) $\quad(B \rightarrow C) \rightarrow((A \vee B) \rightarrow(A \vee C))$.

(A5) $\square A \rightarrow A$.

(A6) $\square(A \rightarrow B) \rightarrow(\square A \rightarrow \square B)$.

(A7) $\diamond A \rightarrow \square \diamond A$.

When $A$ and $B$ are formulas, we suppose the following rules of inference:

(R1) If $\vdash A$ and $\vdash A \rightarrow B$, then $\vdash B$.

(R2) If $\vdash A$, then $\vdash \square A$.

For any formula $A$, we say that $A$ is a classical formula iff $A$ contains none of $\square$ and $\diamond . A\left(x_{1}, \ldots, x_{n}\right)$ denotes a formula, $A$, having exactly $n$ distinct 
proposition variables, $x_{1}, \ldots, x_{n}$, in $\Pi$. When $B_{1}, \ldots, B_{n}$, and $A\left(x_{1}, \ldots, x_{n}\right)$ are formulas, then $A\left(B_{1}, \ldots, B_{n}\right)$ also represents a formula obtained by substituting $B_{1}, \ldots, B_{n}$ for $x_{1}, \ldots, x_{n}$ in $A\left(x_{1}, \ldots, x_{n}\right)$, respectively.

A truth value assignment is a mapping $\mu: \Pi \rightarrow\{0,1\}$, where 0 means false and 1 means true. Let $\Omega$ be the set of all $\mu$ 's. A truth valuation is a mapping $\mu^{*}$ from the set of all formulas into $\{0,1\}$, which is the unique extension of $\mu$ in the following way:

For any formulas $A$ and $B$,

(a) if $A$ is $x_{i}$ in $\Pi, \mu^{*}\left(x_{i}\right)=\mu\left(x_{i}\right)$,

(b) if $\mu^{*}(A)$ and $\mu^{*}(B)$ are defined, $\mu^{*}(A \vee B)=\operatorname{Max}\left\{\mu^{*}(A), \mu^{*}(B)\right\}$,

(c) if $\mu^{*}(A)$ is defined, $\mu^{*}(\sim A)=1-\mu^{*}(A), \mu^{*}(\square A)=\operatorname{Min}\left\{\nu^{*}(A) \mid \nu \in \Omega\right\}$.

We can then easily see that

(d) if $\mu^{*}(A)$ is defined, $\mu *(\diamond A)=\operatorname{Max}\{\nu *(A) \mid \nu \in \Omega\}$.

When $A$ is $A\left(x_{1}, \ldots, x_{n}\right)$, then $\mu *(\square A)$ and $\mu *(\diamond A)$ are actually determined by considering $2^{n}$ cases of $\nu^{*}(A)$ 's for all $n$-tuples $\left(\nu\left(x_{1}\right), \ldots, \nu\left(x_{n}\right)\right) \in\{0,1\}^{n}$, and they take uniformly either 0 or 1 for all cases. A formula $A$ is called valid iff $\mu *(A)=1$ for all $\mu \epsilon \Omega$.

2 Representation of S5 Let $A\left(x_{1}, \ldots, x_{n}\right)$ be a formula of the form $\diamond C \vee \square D_{1} \vee \ldots \vee \square D_{l} \vee E$, where $C, D_{1}, \ldots, D_{l}$, and $E$ are all classical formulas. The following two lemmas are stated:

Lemma 1 If $A\left(B_{1}, \ldots, B_{n}\right)$ is valid for every classical formula, $B_{1}, \ldots, B_{n}$, then at least one of $C \vee D_{1}, \ldots, C \vee D_{l}, C \vee E$ in $A\left(x_{1}, \ldots, x_{n}\right)$ is provable in the classical logic.

Lemma 2 If at least one of $C \vee D_{1}, \ldots, C \vee D_{l}, C \vee E$ in $A\left(x_{1}, \ldots, x_{n}\right)$ is provable in the classical logic, then $A\left(x_{1}, \ldots, x_{n}\right)$ is provable in $\mathrm{S} 5$.

Proof of Lemma 1: Suppose none of $C \vee D_{1}, \ldots, C \vee D_{l}, C \vee E$ is provable in the classical logic. As for classical formulas, truth valuation, $\mu^{*}$, coincides with usual valuation. Hence, $\mu_{i}^{*}\left(C \vee D_{i}\right)=0(i=1, \ldots, l)$, $\mu_{l+1}^{*}(C \vee E)=0$ for some $\mu_{i}^{*}, \mu_{l+1}^{*}$ such that $\mu_{i}\left(x_{j}\right)=e_{i j}, \mu_{l+1}\left(x_{j}\right)=e_{l+1 j}(j=$ $1, \ldots, n)$, respectively. (Each of $e_{i j}$ and $e_{l+1 j}$ is 0 or 1.) We illustrate these relations with the following truth table:

\begin{tabular}{|c|c|c|c|c|c|c|c|c|}
\hline$x_{1}$ & $x_{2}$ & $\ldots \ldots x_{n}$ & $C$ & $D_{1}$ & $D_{2}$ & $\ldots \ldots$ & $D_{l}$ & $E$ \\
\hline$e_{11}$ & $e_{12}$ & $\ldots \ldots e_{1 n}$ & 0 & 0 & & & & \\
\hline$e_{21}$ & $e_{22}$ & $\ldots \ldots e_{2 n}$ & $\begin{array}{l}0 \\
:\end{array}$ & & 0 & - & & \\
\hline$e_{l_{1}}$ & $e_{l_{2}}$ & $\ldots \ldots e_{l n}$ & 0 & & & & 0 & \\
\hline$e_{l+11}$ & $e_{l+12}$ & $\ldots \ldots e_{l+1 n}$ & 0 & & & & & 0 \\
\hline
\end{tabular}

Now, let $k$ be the integer such that $2^{k-1}<l+1 \leqslant 2^{k}$. Take $k$ distinct proposition variables, $y_{1}, \ldots, y_{k}$, in $\Pi$. Define $B_{1}, \ldots, B_{n}$ so as to satisfy 
the next truth table with $2^{k}$ rows, where for the rows from $(l+1)$ 'th to $2^{k}$ 'th, each $B_{j}$ has the same value $e_{l+1 j}(j=1, \ldots, n)$ :

\begin{tabular}{|c|c|c|c|c|}
\hline$y_{1}$ & $y_{2} \ldots \ldots y_{k}$ & $B_{1}$ & $B_{2}$ & $\ldots \ldots B_{n}$ \\
\hline 0 & $0 \ldots \ldots$ & $e_{11}$ & $e_{12}$ & $\ldots \ldots e_{1 n}$ \\
\hline 0 & $0 \ldots 1$ & $e_{21}$ & $e_{22}$ & $\ldots \ldots e_{2 n}$ \\
\hline & $\cdots \cdots$ & $\begin{array}{l}e_{l 1} \\
e_{l+11}\end{array}$ & $\begin{array}{l}e_{l 2} \\
e_{l+12}\end{array}$ & $\begin{array}{l}\ldots \ldots \\
\ldots \ldots e_{l n} \\
\ldots \ldots e_{l+1 n}\end{array}$ \\
\hline 1 & $\begin{array}{c}\ldots \ldots \\
1 \ldots \ldots\end{array}$ & $\dot{e}_{l+11}$ & & $?+$ \\
\hline
\end{tabular}

By the functional completeness of classical logic, $B_{1}, \ldots, B_{n}$ above can be expressed by the disjunctive normal forms having $y_{1}, \ldots, y_{k}$. Then for all $\mu \epsilon \Omega, \mu^{*}\left(C\left(B_{1}, \ldots, B_{n}\right)\right)=0$, i.e., $\mu *\left(\diamond C\left(B_{1}, \ldots, B_{n}\right)\right)=0$. For some $\mu \epsilon \Omega$, $\mu *\left(D_{s}\left(B_{1}, \ldots, B_{n}\right)\right)=0(s=1, \ldots, l)$, hence for all $\mu \in \Omega, \mu *\left(\square D_{s}\left(B_{1}, \ldots, B_{n}\right)\right)=$ 0 . And there exists at least one $\mu \epsilon \Omega$, say $\mu_{0}$, such that $\mu^{*}\left(E\left(B_{1}, \ldots, B_{n}\right)\right)=$ 0 . Hence $\mu_{0}^{*}\left(A\left(B_{1}, \ldots, B_{n}\right)\right)=0$, i.e., $A\left(B_{1}, \ldots, B_{n}\right)$ is not valid. This contradicts the hypothesis.

Proof of Lemma 2: Assume that at least one of $C \vee D_{1}, \ldots, C \vee D_{l}, C \vee E$ is provable in the classical logic. Then it is clearly provable in S5. As for the case $\vdash C \vee D_{s}$, i.e., $\vdash \sim C \rightarrow D_{s},(s=1, \ldots, l)$, we have $\vdash \square \sim C \rightarrow \square D_{s}$, i.e., $\vdash \diamond C \vee \square D_{s}$, by rule (R2), axiom (A6), and rule (R1). Hence $\vdash A\left(x_{1}, \ldots\right.$, $\left.x_{n}\right)$. As for the case $\vdash C \vee E$, we have also $\vdash \square \sim C \rightarrow \square E$, and hence $\vdash \square \sim C \rightarrow E$, i.e., $\vdash \diamond C \vee E$ by (A5). Thus we have again $A\left(x_{1}, \ldots, x_{n}\right)$.

Theorem A formula $A\left(x_{1}, \ldots, x_{n}\right)$ is provable in $\mathrm{S} 5$ iff for every classical formula, $B_{1}, \ldots, B_{n}, A\left(B_{1}, \ldots, B_{n}\right)$ is valid.

Proof: That if $A\left(x_{1}, \ldots, x_{n}\right)$ is provable in S5 then $A\left(B_{1}, \ldots, B_{n}\right)$ is valid for every classical formula, $B_{1}, \ldots, B_{n}$, is clear by verifying that all axioms are valid and all rules of inference preserve validity.

Next, we prove that for a formula $A\left(x_{1}, \ldots, x_{n}\right)$ if $A\left(B_{1}, \ldots, B_{n}\right)$ is valid for every classical formula, $B_{1}, \ldots, B_{n}$, then $A\left(x_{1}, \ldots, x_{n}\right)$ is provable in S5. It is well-known that $A\left(x_{1}, \ldots, x_{n}\right)$ can be reduced in S5 to the modal conjunctive normal form, $A^{\prime}$, which is of the form $A_{1} \wedge \ldots \wedge$ $A_{r}(r \geqslant 1)$, each $A_{\alpha}(\alpha=1, \ldots, r)$ being of the form $\diamond C \vee \square D_{1} \vee \ldots \vee \square D_{l} \vee E$, where $C, D_{1}, \ldots, D_{l}$, and $E$ are all classical formulas, $l \geqslant 0$, and $C$ or $E$ may be missing. Let $B_{1}, \ldots, B_{n}$ be any classical formulas, and suppose $A\left(B_{1}, \ldots, B_{n}\right)$ is valid. Then $A^{\prime}\left(B_{1}, \ldots, B_{n}\right)$ is valid, and so is $A_{\alpha}\left(B_{1}, \ldots, B_{n}\right)$, $(\alpha=1, \ldots, r)$. By Lemma 1 and Lemma $2,{ }^{1}$ we have $A_{\alpha}\left(x_{1}, \ldots, x_{n}\right)$ is provable in $\mathrm{S} 5$, and so is $A^{\prime}\left(x_{1}, \ldots, x_{n}\right)$. Hence $A\left(x_{1}, \ldots, x_{n}\right)$ is provable in S5.

1. If $C$ is missing then $C \vee D_{1}, \ldots, C \vee D_{l}, C \vee E$ degenerate into $D_{1}, \ldots, D_{l}, E$, if $E$ is missing then so is $C \vee E$, and if $l=0$ then $C \vee D_{1}, \ldots, C \vee D_{l}$ are missing. In such special cases, these two lemmas still hold. 
3 Remark We remark that for any (classical) formulas, $B_{1}, \ldots, B_{n}$, $A\left(B_{1}, \ldots, B_{n}\right)$ is valid, iff $A\left(x_{1}, \ldots, x_{n}\right)$ is a tautology of $\mathrm{S} 5$ in the sense of Kripke [2], i.e., iff $A\left(x_{1}, \ldots, x_{n}\right)$ is assigned 1 in every row of every partial truth table of $A\left(x_{1}, \ldots, x_{n}\right)$. In fact, if $A\left(x_{1}, \ldots, x_{n}\right)$ is a tautology, then for any (classical) formulas, $B_{1}, \ldots, B_{n},\left\{\left(\mu^{*}\left(B_{1}\right), \ldots, \mu^{*}\left(B_{n}\right)\right) \mid \mu \in \Omega\right\} \subseteq$ $\{0,1\}^{n}$, hence $A\left(B_{1}, \ldots, B_{n}\right)$ is valid. Conversely, assuming any (classical) formulas, $B_{1}, \ldots, B_{n}, A\left(B_{1}, \ldots, B_{n}\right)$ is valid. We consider any partial truth table, $\sum$, with $m\left(1 \leqslant m \leqslant 2^{n}\right)$ rows of $A\left(x_{1}, \ldots, x_{n}\right)$. Let $k$ be the integer such that $2^{k-1}<m \leqslant 2^{k}$, and take $k$ distinct proposition variables, $y_{1}, \ldots, y_{k}$, in $\Pi$. In the same way as the proof of Lemma 1 , we can construct $B_{j}\left(y_{1}, \ldots, y_{k}\right)(j=1, \ldots, n)$ such that $A\left(B_{1}, \ldots, B_{n}\right)$ satisfies $\sum$. By the assumption, $A\left(B_{1}, \ldots, B_{n}\right)$ is valid. Hence $A\left(x_{1}, \ldots, x_{n}\right)$ is assigned 1 in every row of $\sum$. Therefore, $A\left(x_{1}, \ldots, x_{n}\right)$ is a tautology.

We notice that in the above Theorem and Remark, $B_{1}, \ldots, B_{n}$ do not need to be classical formulas, i.e., they can be any formulas of S5.

In the proof of Theorem 2 of Thomason [3], it was shown that $A$ is valid in S5 (tautology of S5 in the sense of Kripke [2]) iff every formula of $\mathcal{L}_{c}$ of the form $A\left(B_{1}, \ldots, B_{n}\right)$ is valid in $(c$. This fact corresponds with the above remark.

\section{REFERENCES}

[1] Hughes, G. E., and M. J. Cresswell, An Introduction to Modal Logic, Methuen Co. Ltd., London (1968), pp. 116-121.

[2] Kripke, S. A., "A completeness theorem in modal logic," The Journal of Symbolic Logic, vol. 24 (1959), pp. 1-14.

[3] Thomason, S. K., "A new representation of S5," Notre Dame Journal of Formal Logic, vol. XIV (1973), pp. 281-284.

Nagoya Institute of Technology

Nagoya, Japan

and

Toyota Technical College

Toyota, Japan 